\title{
Bioanalysis
}

\section{Stability assessment in ligand-binding assays: a critical parameter for data integrity}

\author{
"...a great deal of confusion remains on how to assess and demonstrate \\ stability in scientifically meaningful ways."
}

Keywords: analyte stability $\bullet$ biotransformation $\bullet$ test article stability

In the last 2 years at the Workshops on Recent Issues in Bioanalysis and the Crystal City $\mathrm{V}$ meetings, many questions regarding stability assessment of analytes have been raised. The questions varied from how the assessments are performed to specific stability related issues. Although the concepts on stability are discussed by regulatory agencies and in industry White papers [1-3], a great deal of confusion remains on how to assess and demonstrate stability in scientifically meaningful ways. While certain specific issues may be difficult to answer, general questions on how to perform stability assessment in ligand-binding assays (LBAs) can be readily answered. These meetings cover topics on both small and large molecule bioanalysis but only some aspects are relevant to both modalities while others may pertain to only one. Understanding how and what to assess for analyte stability is critical in the interpretation of the data for this analyte.

This special issue focuses solely on stability assessments pertaining to large molecule analytes using LBAs in the field of bioanalysis. Stability assessment using LBAs itself is broad and covers multiple aspects; stability of test article in formulation; stability of test article in an intended matrix in support of PK assessment; stability of soluble biomarker proteins in which LBA is used for pharmacodynamics assessment; stability of anti-drug antibody (ADA) in assessment of immunogenicity and; blood sample processing stability of test article(s), and biomarker proteins during sample collection processes.

Stability of test article in formulation is typically performed by either the analytical or formulation division of a company. These divisions will issue certificates of analysis and stability for test articles in formulations stored in their original vials at respective storage temperatures. As long as the bioanalytical laboratories follow these specifications, it is not necessary to reestablish the stability of test articles in formulations. However, stability of the test article should be reexamined if a test article is aliquoted from the originally formulated vial or transferred to a different storage temperature.

The stability of test article in the intended matrix is one of the critical parameters evaluated during prestudy validation. In recent years, there seems to be an ongoing debate on what type of standard calibrators should be used for stability assessments. Bioanalytical laboratories performing LBAs routinely use standard calibrators, which are freshly prepared (less than 3 days) and frozen once in their stability assessments. For large molecule therapeutics (monoclonal antibodies or recombinant proteins), the impact of a single freeze-thaw evaluation is critical. This initial stability assessment is the basis for providing sample collection, storage and handling information to the respective in vivo laboratories and clinics. Once the serum or plasma study samples are collected, they are immediately frozen and shipped to designated bioanalytical sites for measurement of intended analytes. Samples are normally thawed under room temperature (RT) or $2-8^{\circ} \mathrm{C}$ prior to measurement of analytes. In this special issue, Macaraeg et al. have shown how stability assessments for benchtop, freeze-thaw and long-term storage were carried out for

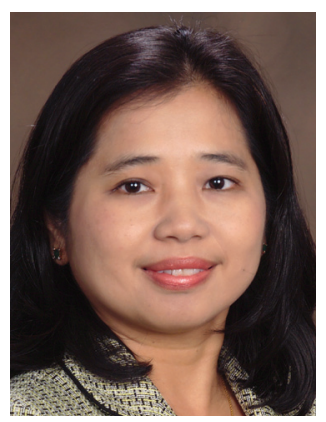

Theingi M Thway Author for correspondence: Department of Pharmacokinetic \& Drug Metabolism, Amgen, Inc., One Amgen Center Drive, Thousand Oaks, CA 91320, USA theingi.thway@amgen.com

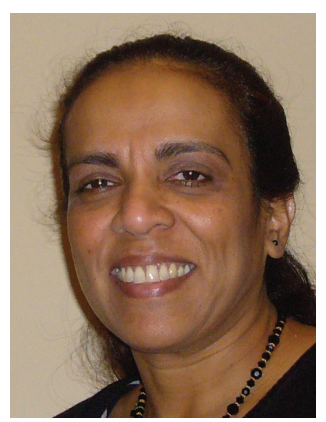

Binodh DeSilva

Department of Immunochemistry \& Biomarker Development, Bristol-Myers Squibb, Route 206 \& Province Line Road, Princeton, NJ 08543, USA 
monoclonal antibody therapeutics and discussed some of the challenges observed [4]. In the approach of Macaraeg et al., recovery of freshly prepared never frozen stability samples were backcalculated using a standard calibrator that was also freshly prepared but went through one freeze-thaw cycle. The intent of this assessment is to validate that the freeze-thaw process has no negative impact on the immunochemical properties of the analyte which could affect analysis in LBA. Once the stability of one freeze-thaw cycle is demonstrated to be within the acceptance criteria, use of standard calibrators that are freshly prepared and frozen is a scientifically justified approach in large molecule bioanalysis.

\section{"...the biotransformation and stability of antibody drug conjugate are interconnected and require special considerations.}

During panel discussions in these meetings, questions on incurred sample stability are often raised. In this special issue, Andisik et al. have demonstrated the long-term storage stability of a monoclonal antibody therapeutic using incurred sample reanalysis [5]. In general, stability of monoclonal antibodies and majority of recombinant protein therapeutics in serum or plasma have not been an issue and thus incurred sample instability has not been a concern in LBA. However, some recombinant protein therapeutics may show proteolytic degradation in vivo. In this case, biotransformation study can provide scientifically meaningful insights. As described in commentary by Rangan et al. [6], the biotransformation and stability of antibody drug conjugate are interconnected and require special considerations. Although the majority of methods for PK assessment are based on LBAs, a few cell-based assays are implemented for PK assessment. In this issue, White et al. demonstrate incurred sample stability assessment in a cell-based PK method, which was designed to measure drug $\mathrm{X}$, a cytokine therapeutic, in a bioequivalent study [7].

Stability of soluble biomarker proteins is covered by Fraser $e t a l$. Development and/or validation of biomarker methods impose distinct challenges relative to methods intended for PK assessment due to the general lack of a 'gold' reference standard [8]. For methods intended for PK assessment, identical materials (test articles) that are used in dosing can be used in the LBAs. In case of biomarkers, recombinant proteins are often used in LBAs for relative quantification of endogenous biomarker proteins in matrices. Due to matrix interference issues, alternative or substituted matrix may be used to prepare the standard calibrators [8]. Thus, it is important to establish the stability of recombinant proteins in either the alternative or substituted matrix to show the stability of standard calibrators prepared for the study. Additionally, the stability of endogenous biomarker proteins in study matrix must be established [9]. Fraser et al. have shown that freeze-thaw or long-term storage stability of two purified recombinant proteins did not reflect endogenous protein stability [10]. Authors reported two cases where; severely compromised measurements of biomarker protein (TGF- $\beta 1$ ) in urine samples from diabetic patients were observed even after one freeze-thaw cycle and; endogenous IL-13 had longer long-term storage stability (15 months) compared with spiked controls using recombinant proteins (4 months). In the case of biomarker proteins, protein characterization data may not be readily available. Yet it is crucial to understand their characteristics since improper folding or unfolding of proteins may impact stability of recombinant proteins and/or their immunochemical properties once spiked into the matrix. The general lack of a 'gold' standard and lack of protein characterization data can limit stability data interpretation of endogenous biomarker proteins.

In contrast to stability of test article in the intended matrix, stability of ADA in assessment of immunogenicity has not been seen as a critical parameter in prestudy validation. As mentioned by Shankar et al., ADAs are polyclonal in nature whether they are against therapeutic proteins $\mathrm{X}$ or $\mathrm{Y}$, and it is reasonable to assume that the stability of ADAs is same for therapeutic proteins $\mathrm{X}$ or $\mathrm{Y}$ in serum or plasma [11]. Therefore, stability of ADA can be approximated by stability of immunoglobulin specific to any antigen in serum or plasma. In addition, the stability assessment using surrogate monoclonal or polyclonal antibodies to determine benchtop, freeze-thaw and long-term storage stability in methods designed to detect ADAs is often seen as a check box exercise with limited scientific value. Now that many companies have their sufficient collections of benchtop, freeze-thaw and longterm storage stability for surrogate ADAs, stability assessment using these positive controls is not routinely performed. In this special issue, the article written by Andisik et al. confirmed the stability of endogenous ADAs using incurred samples for up to 24 months [5].

There is no current consensus in the industry whether assessing blood sample processing stability of test article(s), biomarker proteins and ADAs during sample collection processes should be performed as part of method development or prestudy method validation. In many companies, assessing blood sample processing stability of test article(s), and biomarker proteins is more commonly done as part of method development rather than prestudy validation. Although monoclonal antibodies (mAbs) are known 
for being stable, previously Wang et al. had shown a case where deviation in sample collection times at CRO led to an investigation demonstrating stability of blood sample processing [12]. In this special issue, Andisik et al. have also shown that delayed processing conditions with different durations and temperatures (1.5 h at RT, $3 \mathrm{~h}$ at RT, $3 \mathrm{~h}$ on ice, $6 \mathrm{~h}$ on ice, $10 \mathrm{~h}$ on ice and $10 \mathrm{~h}$ on ice, followed by overnight in a $4^{\circ} \mathrm{C}$ refrigerator) had no impact in blood sample processing stability of a mAb [5]. Nonetheless, more case studies regarding protein therapeutics that are not $\mathrm{mAb}$ could benefit the bioanalytical community.

In addition, Bradford $\mathrm{J}$ also described the CRO perspective of analyte and reagent stability [13] and Wang et al. had provided the overview on current industrial practices and regulatory requirements to assess analyte and reagent stability [14]. The

\section{References}

1 US FDA. Guidance of Industry: Bioanalytical Method Validation. U.S. Department of Health and Human Services Food and Drug Administration, Center for Drug Evaluation and Research Center for Veterinary Medicine, MD, USA (2001). www.fda.gov

2 DeSilva B, Smith W, Weiner R et al. Recommendations for the bioanalytical method validation of ligand-binding assays to support pharmacokinetic assessments of macromolecules. Pharm. Res. 90, 1885-1900 (2013).

3 EMA. Guideline on Validation of Bioanalytical Methods. (2011). www.ema.europa.eu

4 Macaraeg C, Ortiz J, Calamba D et al. Serum sample stability in ligand binding assays: challenges in assessments of long-term, bench top and multiple freeze-thaw. Bioanalysis 7(11), 1361-1370 (2015).

5 Andisik M, DeStefano L, Stefan C et al. Effects of blood processing and sample storage on the stability of biotherapeutic monoclonal antibodies and anti-drug antibodies. Bioanalysis 7(11), 1325-1335 (2015).

6 Rangan V, Myler H, Kozhich K et al. Biotransformation and stability of ADC - payload metabolism and linker cleavage delineation. Bioanalysis 7(11), 1319-1323 (2015).

7 White J, Crossman M, Subramanyam M. Incurred sample reproducibility and stability assessment in a cell-based drug concentration assay. Bioanalysis 7(11), 1347-1353 (2015).

8 Thway TM, Salimi-Moosavi H. Evaluating the impact of matrix effects on biomarker assay sensitivity. Bioanalysis 6(8), 1081-1091 (2014). recommendations on characterization and stability assessment of critical reagents can be referred to in white papers by O'Hara et al. and King et al. [15,16]. Last, the articles in this special issue provide the bioanalytical community with a wealth of information and know-how in assessing stability using LBAs.

\section{Financial \& competing interests disclosure}

T Thway is an employee of Amgen, Inc. and owns Amgen stocks. B DeSilva is an employee of Bristol-Myers Squibb and own the company stocks. The authors have no other relevant affiliations or financial involvement with any organization or entity with a financial interest in or financial conflict with the subject matter or materials discussed in the manuscript apart from those disclosed.

No writing assistance was utilized in the production of this manuscript.

9 Wang J, Lee J, Burns D et al. "Fit-for-purpose" method validation and application of a biomarker (C-terminal telopeptides of type 1 collagen) in Denosumab clinical studies. AAPS J. 11, 385-394 (2009).

10 Fraser S, Fleener C, Ogborne K et al. When close isn't close enough: a comparison of endogenous and recombinant biomarker stability samples. Bioanalysis 7(11), 1355-1360 (2015).

11 Shankar G, Devanarayan V, Amaravadi L et al. Recommendations for the validation of immunoassays used for detection of host antibodies against biotechnology products. JPBA 48(5), 1267-1281 (2008).

12 Wang J, Burns D, Ma M. A systematic approach to investigate the impact of nonclinical blood processing deviations on large-molecule drug bioanalysis. Bioanalysis 4(4), 359-365 (2012).

13 Lefor Bradford J. Analyte and reagent stability in ligand binding assays: a bioanalytical CRO perspective. Bioanalysis 7(11), 1337-1346 (2015).

14 Wang J, Nowatzke W, Ma M. Current industrial practices and regulatory requirements to assess analyte and reagent stability using ligand binding assays. Bioanalysis $7(11)$, 1371-1384 (2015).

15 O' Hara DM, Theobald V, Egan AC et al. Ligand binding assays in the 21st century laboratory: recommendations for characterization and supply of critical reagents. AAPS J. 14(2), 316-328 (2012).

16 King LE, Farley E, Imazato $\mathrm{M}$ et al. Ligand binding assay critical reagents and their stability: recommendations and best practices from the Global Bioanalysis Consortium Harmonization Team. AAPS J. 16(3). 504-515 (2014). 